\title{
Mentoría entre pares en la educación médica de pregrado como herramienta para mejorar el aprendizaje y responder a las demandas de las nuevas generaciones
}

\section{Peer mentoring in pregraduate medical education as a tool for improving the learning proccess and satisfy new generation requirements}

Correspondencia

Edali Gloria Ortega Miranda Alameda Julio C. Tello Nro. 231, Dpto. 404, Torres de San Borja. San Borja - Lima.

Teléfono: +511954052222

edaligloria@gmail.com

Recibido: 27/12/2019

Arbitrado por pares

Aprobado: 27/02/2019

Citar como: Ortega-Miranda EG. Mentoría entre pares en la educación médica de pregrado como herramienta para mejorar el aprendizaje y responder a las demandas de las nuevas generaciones. Acta Med Peru. 2019;36(1):57-61

\author{
Edali G. Ortega-Miranda 1,2ab \\ 1 Hospital Guillermo Kaelin de la Fuente. Lima, Perú \\ 2 Universidad Peruana Cayetano Heredia. Lima, Perú. \\ a Médico especialista en Pediatría y Medicina General Integral, b magister en Atención integral al Niño
}

\section{RESUMEN}

Este ensayo se centra en la mentoría aplicada a la enseñanza de pregrado. Se buscará demostrar que la mentoría se fortalece con las tecnologías de la información (TICS) y es útil para los estudiantes que actualmente cursan pregrado, conformado por las generaciones millenials $y$ centenials. Estas generaciones provocan retos y desafíos que constituyen oportunidades para la integración de las tecnologías de las TICs al proceso educativo. La mentoría entre pares, en la que el mentor puede ser un estudiante de años superiores con ciertos requisitos, constituiría una herramienta útil para permitir una mayor adhesión a la carrera, la integración natural de las TICs a la educación, así como un aprendizaje y aprehendizaje más significativos.

Palabras clave: Mentoría; Educación de pregrado en Medicina; Tecnologías de la información (fuente: DeCS BIREME).

\section{ABSTRACT}

This paper is focused in mentoring in pregraduate medicine teaching. The idea is to show that mentoring is strengthened with information technology and that it is useful for pregraduate students, a population mainly comprising millennials and centennials. These generations represent challenges that may be turned into opportunities for incorporating information technology in their educational process. Peer mentoring, in which mentors may be senior students complying with certain requisites, may become an useful tool for achieving greater adhesion to their careers, by naturally integrating information technology to the educational process, leading to more fruitful learning and comprehension.

Keywords: Mentorship; Education, Medical, undergraduate, Information technologies (source: MeSH NLM). 


\section{INTRODUCCIÓN}

La mentoría, o mentoring, constituye una inveterada forma de transmisión de conocimientos usada en todas las áreas del saber humano. El término alude a Mentor, personaje de La Odisea a quien Ulises encarga la educación de su hijo Telémaco. Aunque en término es usado en ocasiones como sinónimo de maestro o tutor, existen diferencias que deben tenerse en cuenta ${ }^{[1]}$.

La mentoría es un proceso en el cual una persona con experiencia -el mentor- aconseja, guía y da soporte profesional y personal a una persona con potencial elevado -el mentorizado- con el fin de que este último logre desarrollar su talento ${ }^{[2]}$, todo en un ambiente de respeto mutuo, confianza y diálogo. La mentoría está asociada positivamente con la permanencia y satisfacción en la carrera así como con mejor producción científica ${ }^{[3-5]}$.

El presente ensayo se enfoca en la mentoría aplicada a la enseñanza de pregrado. Se buscará demostrar que la misma no está divorciada de las actuales Tecnologías de Información y comunicación (TICS) ni de las características de los estudiantes a los que va dirigido el mismo (millenials y centenials) sino que, por lo contrario, se ve fortalecida con una serie de oportunidades a explotar.

Iniciaré discutiendo acerca de la mentoría en la enseñanza de pregrado de medicina para luego tratar acerca de los retos multigeneracionales e informáticos a los que enfrenta la mentoría y la enseñanza médica del siglo XXI. En cada uno de estos acápites se darán argumentos que defienden el uso de la mentoría como herramienta activa y positiva para el proceso enseñanza-aprendizaje y cómo el mismo puede potenciarse con el uso de las TICs.

\section{La mentoría en la enseñanza de pregrado de medicina}

La medicina, según plantea Lifshitsz ${ }^{[1]}$, se aprende según algunos preceptos: a) aprender haciendo, b) motivación basada en la responsabilidad, c) supervisión y asesoría, d) vinculación teoríapráctica, e) ambiente académico, f) investigación como estrategia educativa, y g) experiencias significativas. Son estos momentos los que deben potenciarse y volverse más significativos para garantizar la formación de excelencia en medicina, y ello podría promoverse con la mentoría.

Es importante distinguir entre dos momentos en la educación del estudiante de medicina: los estudios preclínicos y los años de estudios clínicos propiamente dichos. Los estudios preclínicos son el primer acercamiento del estudiante a la Medicina; durante este tiempo cobran importancia, además de las lecciones impartidas, el comportamiento, lenguaje extraverbal y actitudes del docente médico, por ende, el modelo a seguir, tanto positivo como negativo. Durante los años de estudios clínicos, el estudiante tiene un mayor contacto con el paciente y se vislumbran mejor sus propias aptitudes y actitudes; en esta etapa serían claves los modelos a seguir y los mentores, pues los ayudarán a definir mejor el área de trabajo que querrán seguir en su futura vida profesional ${ }^{[6]}$.

Tradicionalmente, la mentoría está asociada a una relación "vertical" entre una persona de mayor conocimiento y una en aprendizaje. Por esto, se entiende históricamente como mentores a aquellos docentes o médicos ya graduados, especialistas la mayoría de las veces; no obstante, también es posible una modalidad "horizontal" -simétrica o entre pares- en la que el mentor y el mentorizado se encuentran en situaciones similares, donde el mentor puede ser un estudiante de años superiores que cumple ciertos requisitos ${ }^{[7]}$

La mentoría entre pares promueve un clima de mayor confianza y comprensión gracias al plano de igualdad entre el mentor y mentorizado, así como las características que comparten (problemas y necesidades similares, experiencias académicas recientes, empatía con sus iguales, ayuda mutua, etc.). El mentorizado podrá ser aconsejado, orientado, motivado y ayudado en el qué hacer y cómo hacerlo, así como en el desarrollo de estrategias de trabajo intelectual y aprendizaje. Para ello, el compañero-mentor debe ser un estudiante calificado y con disponibilidad de tiempo para dedicarlo a su mentorizado. Este tipo de mentoría debería estar supervisado por un consejero o mentor de más rango (docente) que pueda guiar el proceso de forma adecuada ${ }^{[7]}$.

Aunque los estudios que evalúen el efecto de la mentoría aún son escasos y poco concluyentes, sus resultados son positivos. Sukreja et al. ${ }^{[8]}$ reportaron resultados satisfactorios en un programa piloto de mentoring dirigido a estudiantes de primer año; los mentores entrevistados indicaron que el programa fue útil para sus labores de enseñanza y habilidades de comunicativa, los estudiantes que los había ayudado emocional y académicamente.

Fallatah et al. ${ }^{[9]}$ realizaron un estudio que buscaba establecer la relación entre las características del mentor y el rendimiento de los estudiantes dentro de un curso de habilidades clínicas, con el fin de identificar a aquellos que requirieran un mayor soporte. Concluyeron que el $83 \%$ de los estudiantes consideraba a la mentoría era importante para ellos, aunque no tuvo un efecto significativo en su desempeño académico. Esto último podría deberse al tiempo de mentoría, que duró sólo una rotación, como al hecho de que se tenía un mentor por cada diez mentorizados. Tiempo y trabajo personalizado son dos elementos a tener muy en cuenta en el proceso de mentoría.

Se concibe a la mentoría como un proceso a mediano y largo plazo, por lo que sus frutos no pueden ser evaluados en un periodo corto de tiempo; así como que debe ser un trabajo personalizado, incluso individualizado, para que haya la oportunidad de establecer un ambiente de confianza y diálogo entre mentor y mentorizado y la experiencia pueda resultar mutuamente significativa.

Ho et al. ${ }^{[10]}$, en un estudio realizado en Korea, concluyeron que la satisfacción de los estudiantes con la mentoría se asociaba 
tanto al año académico del estudiante como a la frecuencia de reuniones y los temas a tratar. Nuevamente, tiempo y trabajo personalizado, traducido en frecuencias necesarias para que el estudiante se sienta motivado y en confianza, así como la suficiente individualización del proceso para que se puedan desarrollar los temas que son importantes para el estudiante según sus necesidades y expectativas.

En el marco anteriormente mencionado, la mentoría en pares, adecuadamente organizada y supervisada, puede resultar útil y beneficiosa para los estudiantes de reciente ingreso. Akinla et al. ${ }^{[11]}$, en una revisión sistemática sobre los resultados de programas de mentoría por pares dirigidas a estudiantes de primer año de la carrera, encontraron que tener mentoría se relacionaba con disminución del estrés, mayor facilidad de adaptación e identificación con el desarrollo personal y profesional. Asimismo, favorecía al desarrollo de actitudes personales y profesionales en los estudiantes mentores. No obstante, la calidad de sus métodos de evaluación fue de bajo a moderado, por lo que se requieren más estudios.

La mentoría por pares, además, ofrece la ventaja de ser un proceso mucho más personalizado; por otro lado, como se ha mencionado, este proceso también es beneficioso para el mentor, puesto que le ayuda a consolidar sus conocimientos y habilidades médicas, así como a desarrollar una mayor empatía, necesaria para el trabajo en equipo, fundamental en cualquier área de la salud. En tal sentido, el establecimiento de programas formales, con la supervisión de un docente de este proceso, sería una opción de enriquecimiento y fortalecimiento del proceso enseñanza - aprendizaje.

\section{Retos multigeneracionales e informáticos para} la mentoría y la enseñanza médica del siglo XXI

La medicina y la enseñanza médica del siglo XXI tienen un escenario interesante y variado en cuanto a generaciones, formas de trabajo y métodos de aprendizaje respecta. Algunos estudios como el de Mohr et al. ${ }^{[12]}$ y el de Boysen et al. ${ }^{[13]}$ hacen hincapié en el hecho de que la educación médica actual cuenta con varias generaciones, diferentes en varios aspectos: los tradicionalistas, los baby boomers, la generación $\mathrm{X}$, la generación $\mathrm{Y}$ o millenials $\mathrm{y}$ la generación Z o centenials.

Los primeros, nacidos antes de 1945 , se describen como dedicados, convencionales, respetuosos del orden y la autoridad, y altruistas; conciben a la medicina más como vocación que como trabajo, pocos se mantienen aún en actividad, generalmente dirigiendo los departamentos sanitarios o son profesores eméritos y un referente para el resto de médicos ${ }^{[12,13]}$.

Los segundos, nacidos entre 1945 y 1964, creen en el trabajo duro, llegando temprano y yéndose tarde, aspiran a mejores compensaciones económicas, acordes al esfuerzo y desempeño, consideran que las promociones en cargos deben ganarse con el tiempo y apreciando el reconocimiento y los puestos directivos; por ello, valoran las cadenas de mando y pueden ser muy críticos con puntos de vista diferentes a los suyos ${ }^{[12,13]}$.

La generación X, nacidos entre 1964 y 1980, son considerados independientes, pragmáticos y flexibles, leales consigo mismos $y$ sus familias antes que con las instituciones. No dudan en cambios laborales si estos les ofrecen mejores perspectivas. Cuestionan la autoridad y prefieren ser evaluados por sus logros que por el tiempo en el trabajo. Al haber nacido y crecido en medio del auge de la computación, les resulta más fácil adaptarse a la tecnología que a las generaciones anteriores, son autodidactas y pueden asimilar información rápidamente cambiante, así como resolver problemas con facilidad y participar en experiencias de aprendizaje cooperativo $^{[12,13]}$.

Los millenials, nacidos entre 1980 y 1999, son optimistas, colaborativos, aprecian el trabajo en equipo y conocen muy bien la tecnología. Desean resultados rápidos, llegando a ser impacientes. Tienen múltiples intereses además de la medicina. Les gusta el reconocimiento y la recompensa, así como estar conectados con otros, sobre todo con sus colegas. Se sienten comprometidos con la salud global. Desean retroalimentación y tienen tendencia a esperar elogios. Tienen gran capacidad de organización y movilización, y se les da bien el trabajo en equipo y seguir las reglas. Les gusta expresar sus opiniones y que estas se tomen en cuenta. Esperan horarios de trabajo flexibles que les permitan un balance adecuado entre su vida personal y laboral. Al estar expuestos a la tecnología toda su vida, desean tenerla siempre a la mano y prefieren las fuentes electrónicas a las físicas para su aprendizaje. En este grupo se encuentra actualmente la mayoría de estudiantes de medicina, residentes y especialistas jóvenes ${ }^{[12,13]}$.

Los centenials, o generación 2020, nacidos luego de 1997, son más hiperconectados y les resulta más sencillo el uso de computadoras y de internet que a los millenials. Tienen una visión del mundo donde la tecnología tiene un rol central, con expectativas por oportunidades de educación global y aprendizaje a través de experiencias y el trabajo en pares. Sus teléfonos móviles los ayudan en todas sus actividades, tanto las personales como educativas. Prefieren estar conectados que recibir una educación formal en un aula. Son los estudiantes de primeros años de la carrera, lo que representa todo un reto respecto a las formas de enseñanza a aplicar con ellos ${ }^{[13]}$.

La descripción hecha de cada uno de estos grupos generacionales sirve para entender que la forma de enseñanza de la medicina en los últimos 70 años, desde la segunda mitad del siglo XX, ha cambiado de forma notable. De los tratados y los libros hemos pasado a las revistas electrónicas. Los artículos científicos que antes llegaban por correo ahora están a la distancia de un clic. La forma de establecer interrelaciones también ha cambiado, muchos jóvenes de las dos últimas generaciones han llegado a sentirse más ligados e identificados con personas que se encuentran a miles de kilómetros de distancia que con aquellas que comparten un mismo entorno geográfico. Esto establece una serie de desafíos pero también la oportunidad de enriquecer 
la enseñanza de la medicina: no perder su esencia personal y humana, pero sí globalizarla, permitir que las TICs tomen un rol protagónico y de esa manera los actuales estudiantes de medicina, multifuncionales, hiperconectados, puedan aprender entre la tradición y la modernización.

Las TICs se definen de muchas maneras; según Cabero son aquellas "... que giran en torno a tres medios básicos: la informática, la microelectrónica y las telecomunicaciones; pero giran, no sólo de forma aislada, sino lo que es más significativo de manera interactiva e interconectada, lo que permite conseguir nuevas realidades comunicativas" ${ }^{[14]}$.

El impacto de las TICs en la educación superior depende de muchos factores. Nakano et al. ${ }^{[15]}$ concluyen que la incorporación de las TICs requiere tanto el conocimiento y dominio de las herramientas como el enfoque pedagógico orientado a ellas, también el nivel de competencias digitales de docentes y estudiantes. Esto es importante si tenemos en cuenta las brechas generacionales de los docentes médicos y los actuales estudiantes de pregrado.

Por otro lado, no puede olvidarse que nuestro país es un país de grandes inequidades que se hace extensivo al acceso a las TICs. En el informe del Instituto Nacional de Estadística e Informática del primer trimestre de $2018^{[16]}$ se observa que mientras que el $70,9 \%$ de la población de Lima Metropolitana tiene acceso a internet, el porcentaje disminuye en el resto de áreas urbanas $(55,6 \%)$ y en áreas rurales $(13,2 \%)$; lo que debe tenerse en cuenta al planificar una educación adecuada para las actuales generaciones de estudiantes universitarios.

Todo lo mencionado permite realizar el siguiente planteamiento: la mentoría, y de forma especial la mentoría de pares, es una gran oportunidad para poder guiar, apoyar y consolidar al actual estudiante de medicina en su elección de carrera y en su vida universitaria.

Al pertenecer los mentores-compañeros a una generación similar y por ende ser millenials, les resulta más sencillo interrelacionarse con otros millenials e incluso centenials debido a que comparten características similares, habilidades análogas e intereses comunes. Tienen además la posibilidad de comunicarse de forma continua a través de las TICs, herramienta que utilizan de forma cotidiana, lo que facilita la mentoría individualizada. Les permite también mayor facilidad en cuanto a la supervisión por parte de un consejero y es una apuesta por la que deberían decantarse las facultades de medicina: permitir que los nativos informáticos guíen a los propios nativos informáticos y faciliten la comunicación entre ellos con generaciones de migrantes informáticos (tradicionalistas, baby boomers, generación X), que deben adecuarse a un mundo interconectado y a estudiantes de medicina que esperan que este mundo interconectado sea parte activa y vital para la enseñanza y aprendizaje de la medicina.

Esto, por supuesto, va a estar circunscrito a la realidad de cada facultad de medicina y cada universidad, su equipamiento y su presupuesto; pero sin duda cada casa de estudios debe considerar dentro de su ecosistema educativo la adecuada implementación de las TICs, las que, como se ha desarrollado, unidas al mentoring pueden facilitar el aprendizaje significativo y el aprehendizaje en la carrera de medicina.

\section{CONCLUSIONES}

La enseñanza de medicina en pregrado enfrenta grandes retos debido a las diferencias generacionales y a los cambios del mundo moderno. Estos retos y desafíos constituyen oportunidades de integración entre las diversas generaciones y de introducción de las TICs en el currículo educativo; y en estos procesos, la mentoría, de forma particular la mentoría por pares, puede resultar una herramienta útil y necesaria para permitir una mayor adhesión a la carrera por estudiantes de nuevo ingreso, así como un aprendizaje y aprehendizaje médico más significativo.

\section{Fuentes de financiamiento: Recursos propios.}

Conflictos de interés: Ninguno.

\section{REFERENCIAS BIBLIOGRÁFICAS}

1. Lifshitz Al. Mentores [editorial]. Med Interna Méx. 2017;33(2):147-9.

2. Krishnan RJ, Uruthiramoorthy L, Jawaid N, Steele M, Jones DL. Mentoring needs of distributed medical education faculty at a Canadian medical school: a mixed-methods descriptive study. Can J Rural Med. 2018;23(3):68-75.

3. Benson CA, Morahan PS, Sachdeva AK, Richman RC. Effective faculty preceptoring and mentoring during reorganization of an academic medical center. Med Teach. 2002;24(5):550-7. doi: 10.1080/0142159021000002612

4. Sambunjak D, Straus SE, Marusić A. Mentoring in academic medicine: a systematic review. JAMA 2006;296(9):1103-15. doi: 10.1001/jama.296.9.1103

5. Sambunjak D, Straus SE, Marusić A. A Systematic review of qualitative research on the meaning and characteristics of mentoring in academic medicine. J Gen Intern Med. 2010;25(1):728. doi:10.1007/s11606-009-1165-8

6. Denton GD, Hemmer PA. Mentoring and fostering professionalism in medical students: from the classroom to the clerkship. En: Humphrey HJ. Mentoring in Academic Medicine. Philadelphia, Pennsylvania: American College of Physicians Press; 2010. p. 85-103.

7. Manzano N, Martín A, Sanchez M, Rísquez A, Suárez M. El rol del mentor en un proceso de mentoría universitaria. Educación XXI. 2012;15(2):93-118. doi: 10.5944/educxx1.15.2.128

8. Kukreja S, Chhabra N, Kaur A, Arora R, Singh T. Introducing mentoring to 1st-year medical students of a private medical college in North India: a pilot study. Int J Appl Basic Med Res. 2017;7(Suppl 1):S6771. doi:10.4103/ijabmr.IJABMR_160_17

9. Fallatah $\mathrm{HI}$, Soo Park Y, Farsi J, Tekian A. Mentoring clinicalyear medical students: factors contributing to effective mentoring. J Med Educ Curric Dev. 2018;5:2382120518757717. doi:10.1177/2382120518757717

10. Ho Y, Kwon OY, Park SY, Yoon TY. A study of satisfaction of medical students on their mentoring programs at one medical school in Korea. Korean J Med Educ. 2017;29(4):253-62. doi:10.3946/ kjme.2017.71 
11. Akinla $\mathrm{O}$, Hagan $\mathrm{P}$, Atiomo W. A systematic review of the literature describing the outcomes of near-peer mentoring programs for first year medical students. BMC Med Educ. 2018;18(1):98. doi:10.1186/ s12909-018-1195-1. Erratum en: BMC Med Educ. 2018;18(1):167. doi: 10.1186/s12909-018-1273-4

12. Mohr NM, Moreno-Walton L, Mills AM, Brunett PH, Promes $S B$, et al. Generational influences in academic emergency medicine: teaching and learning, mentoring, and technology (part I). Acad Emerg Med. 2011;18(2):190-9. doi:10.1111/j.15532712.2010.00985.x

13. Boysen PG 2nd, Daste L, Northern T. Multigenerational Challenges and the future of graduate medical education. Ochsner J. 2016;16(1):101-7.
14. Belloch C. Las tecnologías de la información y comunicación (T.I.C.) [internet]. Valencia, España: Unidad de Tecnología Educativa, Universidad de Valencia; 2009 [citado 10 nov 2018]. Disponible en: https://www.uv.es/ bellochc/pdf/pwtic1.pdf

15. Nakano T, Garret P, Vásquez A, Mija A. La Integración de las TIC en la educación superior: reflexiones y aprendizaje a partir de la experiencia PUCP. En blanco \& Negro. 2014;4(2):65-76.

16. Ruiz R, Castro Z. Estadísticas de las tecnologías de la información y organización en los hogares, enero-febrero-marzo 2018, No 2 [internet]. Lima, Perú: Instituto Nacional de Estadística e Informática; 2018 [citado 10 nov 2018]. Disponible en: https:// www.inei.gob.pe/media/MenuRecursivo/boletines/01-informetecnico-n02_tecnologias-de-informacion-ene-feb-mar2018.pdf

\title{
Las ediciones anteriores de Acta Médica Peruana están disponibles en:
}

\author{
www.redalyc.org
}

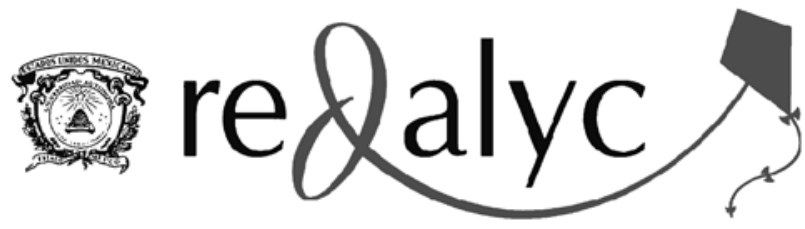

